\title{
Drosophila Hox genes induce melanised pseudo-tumours when misexpressed in hemocytes
}

Running title: Hox genes are causally linked to leukaemia in Drosophila

Titus Ponrathnam and Rakesh K Mishra*

${ }^{1}$ CSIR-Centre for Cellular and Molecular Biology, Hyderabad, Telangana, 500007, India

* To whom correspondence should be addressed. Tel: +91-40-27192600; Fax: +91-40-

27160591; Email: mishra@ccmb.res.in

Keywords:

Hemocytes

melanised pseudotumours

acute myeloid leukaemia

leukaemia/leukemogenicity

haematopoiesis

Hox genes 


\begin{abstract}
:
Homeotic genes are the key early determinants of cell identity along the anterior-posterior body axis across bilaterians. More recently, however, several late non-homeotic functions of hox genes have emerged in a variety of organogenesis processes, including in mammals. Being crucial factors in determining cell identity and organogenesis, the misregulation of hox genes is likely to be associated with defects in these processes. Several studies have reported misexpression of hox genes in a variety of malignancies including acute myeloid leukaemia. Considering that Drosophila is a well-established model for the study of haematopoiesis, we ectopically expressed the hox genes, $D f d, U b x, a b d-A$ and $A b d-B$, to ask if and how it will alter the process of haematopoiesis. We observed black melanised spots circulating in the viscera of the larvae and extensive lethality at during the pupal stage in these conditions. Such abnormalities are the hallmark of dysregulated haematopoiesis. We also observed an increase in blood cell number as well as their enhanced differentiation into lamellocytes. Our study opens a new possibility of addressing the function hox genes in normal and leukemogenic hematopoiesis with potential implications in downstream targets for diagnostic markers and therapy.
\end{abstract}


Summary: Drosophila Hox genes, when expressed in blood cells, are leukemogenic, induce cell autonomous proliferation and differentiation. This reinforces previous studies in vertebrates and allows for Hox induced leukaemia to be studied in Drosophila. 


\section{Introduction:}

One of the most striking aspects of life is the variety in body forms. Despite this variety, there is an even more striking similarity at the genetic and molecular level, in the developmental mechanisms that lead to variety across the species. For example, in spite of the evolutionary distance of hundreds of millions of years between vertebrates and Drosophila, many organ and tissue types show a degree of homology with each other and much of the key developmental pathways are highly conserved.

The hematopoietic system is no exception to this conservation. Hemocytes of Drosophila resemble the myeloid lineage of blood cells [1]. The most abundant cells, plasmatocytes, are the equivalent of macrophages that are involved in a variety of processes, such as responses to pathogens, removal of apoptotic cells, deposition of the extracellular matrix during embryonic development, etc. [2]. The next most abundant cells are the Crystal cells, specialised to induce melanisation reactions in the presence of pathogens and wound healing [3], resemble the granulocytes, and contribute about four per cent of the blood cells. Lamellocytes are the least abundant population of blood cells, usually only appearing in circulation upon the larva being challenged by any object too large to be cleared off by the macrophages, for example, parasitoid wasp eggs [4,5].

The extent of this resemblance includes the molecular pathways involved in the development of these cell types. Serpent, [6] is related to GATA 1, 2 and 3 of vertebrates. GATA-2 is responsible for blood progenitor proliferation and survival [7,8]. GATA-1 is required for progenitor differentiation into erythrocytes, megakaryocytes and eosinophils [9-11]. Similar to GATA-2, $\operatorname{Srp}$ is required for progenitor maintenance and proliferation. Loss of function in Srp leads to a reduced number of progenitors and a loss of all hemocytes. It is also required in plasmatocyte differentiation, similar to GATA-1 [12]. Additionally, ushaped is related to the Friend of GATA (FOG) family. FOG-1 and GATA-1 are required together for erythrocyte and megakaryocyte differentiation [13,14]. FOG-1 interacts with GATA-1 to represses eosinophil differentiation and must be downregulated for eosinophil differentiation [15]. Similarly, ush is expressed in hemocyte precursors and plasmatocytes, and must be downregulated for crystal cell development [16].

Various signalling pathways involved in regulating hematopoiesis, as would be anticipated, are conserved between vertebrates and Drosophila. Examples include Jagged-1, the vertebrate homolog of Serrate, a ligand of Notch, is produced by the stromal cells of the bone 
marrow, to regulate HSC proliferation and survival [17]. Ser performs a similar role, being secreted by cells of the Posterior Signalling Centre [18], a set of regulatory cells at the posterior end of the Lymph Gland (LG). Vertebrate JAK2 is required for erythropoiesis [19], while STAT5 is required for proper progenitor and myeloid cell function [20]. The Drosophila JAK/STAT pathway is required within the LG for the maintenance of prohemocytes, among other things [21]. Transformations in JAK2 can lead to leukemogenesis in vertebrates [22], similar to how gain of function hop mutants behave [23]. The Toll pathway is also conserved, playing a major role in innate immunity in both vertebrates and flies [24].

One aspect of vertebrate hematopoiesis that has not been mirrored in Drosophila is the role of Hox genes. Hox genes are well known for their conserved role in body axis formation across all bilaterians[25], but also play roles in vertebrate hematopoiesis [26], autophagy [27], as well as cell proliferation, differentiation, migration and apoptosis [28]. Hox genes are transcribed in HSCs as well as lineage progenitors, while being suppressed in differentiated blood cells [29-33]. Overexpression models lead to blockages in certain stages of development, expansion of HSCs, the circulation of blast cells, etc. [34-39]. On the other hand, in Drosophila, other than Antp, which is implicated in setting up the location of the LG [40], as well as later marking the PSC [41] hox genes are not known to play any major role.

In this study, we show that overexpression of hox genes, $D f d, U b x, a b d-A$ and $A b d-B$, in the blood cells not only leads to melanised bodies, but also to a significant increase in blood cell number and the induction of lamellocyte differentiation. These findings have implications in understating the biological events associated with leukaemia in humans which may open new possibilities of markers and therapy.

\section{Material and methods:}

Fly culturing: Flies were cultured in standard cornmeal and sucrose agar. The wild-type flies used in this study were Canton-S. Flies were maintained at $25^{\circ} \mathrm{C}$. The Gal4 driver lines used in this study were obtained from the Bloomington stock centre. The UAS lines used in this

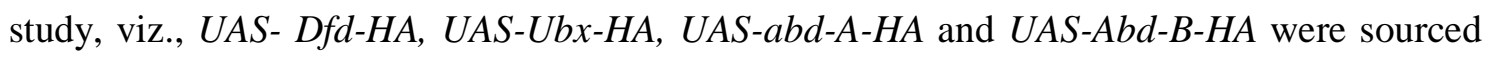
from Yacine Graba's lab. Flies were allowed to lay eggs for 6 hours before being transferred to a fresh vial. Larvae were sacrificed at 96-102 hours post egg lay, before the onset of metamorphosis. 
Immunostaining: For staining proliferative cells we made use of Anti-PhosphoHistone 3 at serine 10, from Upstate (cat\# 07-212, 1ng/ $\mu \mathrm{L}$ ). For confirming the presence of lamellocytes, we used anti-myospheroid (DSHB \#CF.6G11, 27pg/ $\mu \mathrm{L}$ ). Blood cells were prepared using an established protocol [42]. Blood cells numbers were quantified using a modified version of the protocol by Petraki, Alexander, \& Bruckner, 2015 [43].

\section{Results:}

When we expressed the UAS lines different hox genes, $D f d, U b x, a b d-A$ and $A b d-B$, under two hemocytes specific drivers, $\mathrm{He}$-Gal4 and $\mathrm{Hml}$-Gal4, we observed melanised spots. Such melanised spots are absent when a driver specific to the fatbody, Lsp2-Gal4, is used but found when a driver expressing in both the fatbody and hemocytes, cg-Gal4, is used. We also observed the degree of penetrance is in proportion to the strength of the respective drivers in hemocytes (Figure 1). Taken together, this implied that the melanised pseudo-tumour phenotype we observe is of hemocyte origin.

We quantified the number of blood cells in our overexpression lines using a modified version of established methods [42,43]. Larvae were dissected in fixed volumes of PBS in $8 \mathrm{~mm}$ chambers; blood cells were allowed to settle, fixed and then visualised with phalloidin and made a relative estimation of the number of blood cells in each larva by imaging five fields of view and summing up the number (Figure 2). Our results show that when under the expression of the fatbody driver $L s p 2$, the number of hemocytes was comparable to the controls. Blood specific expression, however, led to a significant increase in the number of circulating hemocytes. Interestingly, while the penetrance of melanised spots was lower, blood specific drivers showed a larger number of blood cells (Figure 2).

Previous studies have shown that cells of the LG do not enter into circulation until the onset of metamorphosis. However, $\mathrm{Hml}$ and $\mathrm{cg}$ express in the cortical region of the $\mathrm{LG}$, and $\mathrm{He}$ expresses throughout. Thus, the question arose as to whether the increase in cell number was due to an increase in cell proliferation at the LG or were circulating cells proliferating in a cell-autonomous manner. To this end, we checked for the presence of the mitotic cell marker PH3. Again, we observed cells positive for PH3, when Hox genes were expressed in the blood cells, and not when expressed exclusively in the fatbody (Figure 2, Supplementary Figure 1). Unlike previous reports, we did not find proliferative cells in our controls [44]. This may be due to a loss of cells in our preparations or more robust immunostaining on our 
part. Thus, while we cannot rule out the possibility that LG cells contribute to this increase, at least a fraction of the increase takes place due to the cell autonomous division of Hox overexpressing cells.

While imaging the blood cells, we noticed that there were larger, flattened cells in circulation, reminiscent of lamellocytes. We wished to know if these were bona fide lamellocytes, to which end we stained blood cells for the lamellocyte marker myospheroid (Figure 3, Supplementary Figure 2). In control larvae, there were none, or if present, a handful of lamellocytes in circulation. In our overexpression lines, however, we noticed that not only were lamellocytes mys+, but so were some of the plasmatocyte like cells. None of the plasmatocytes in the control flies or those overexpressing $A b d-B$ showed mys. Thus, we can speculate that these cells, upon Hox overexpression, are pushed toward the lamellocyte fate.

We also noticed that many pupae were not eclosing when the $c g$ driver was used. It has previously shown that aberrant blood cells can induce pupal lethality [45]. However, while we did observe some pupal lethality when the Hox genes were expressed under $\mathrm{He}$ and $\mathrm{Hml}$, the greatest recapitulation of this lethality was when the Lsp2 Gal4 driver was used (Figure 4). This may be on account of a previous result that suggests Hox genes are repressors of autophagy [27].

\section{Discussion:}

We show that the four Hox genes used in this study in Drosophila are capable of inducing melanised bodies in circulation when expressed in the blood cells. These melanotic spots appear only when expressed with Gal4 driver lines active in the LG and circulating blood cells. These spots, therefore, are likely to be aggregations of hemocytes mounting an ectopic immune response. The expression of the genes also triggers cell proliferation. The cells appear to divide in a cell-autonomous manner, which is reflected in the detection of PH3+ cells in circulation. The presence of myospheroid positive elongated cells, seen in circulation, also suggests that Hox overexpression leads to the differentiation of the circulating blood cells into lamellocytes. While overexpression of $a b d-A$ shows relatively stronger phenotypes described here, $A b d-B$ overexpression does not. It supports our earlier finding in which we observed a non-homeotic growth promoter role of $a b d-A$ during formation of the adult cuticle during pupation, while $A b d-B$ does not show any such role [46].

Our results indicate that Hox genes are causal in leukaemia, reinforcing previous studies in vertebrate model systems, and extending these findings to Drosophila. This also opens the 
possibility that Hox gene induced leukaemias, especially those of the myeloid lineage, can be studied and modelled in Drosophila. Till date, the only known Hox gene to participate in hematopoiesis is Antp, which marks in the PSC, and provides spatial signals for the development of the LG. In vertebrates, Hox genes have been shown to express within progenitor cells and are rapidly switched off during cell maturation. As our overexpression lines perturb both cell number and differentiation, it is likely that multiple Drosophila Hox genes are involved in finetuning the precise programme of Drosophila blood cell development.

The fact that the cells appear to be phenotypically confined to plasmatocytes and lamellocytes implies that expression of these genes works in tandem with, and above the specific programme of the cell types. It would be interesting to know which genes are being modulated in our overexpression lines, by profiling their transcription states as well as the binding sites of the individual Hox proteins. In the absence of this information, we speculate that Hox gene overexpression leads to the aberrant transcription of genes. It is known from previous studies that Hox dysregulation in leukaemia is usually concomitant with gain or loss of function mutations in upstream regulators, most commonly Mixed Lineage Leukemia-1 (MLL-1) fusion proteins [47,48], or loss of function EZH2 mutations [49]. It has been reported that MLL-1 fusion proteins have the lowest number of co-operating mutations to induce leukemogenesis [50]. Taken together, we speculate that these driver mutations induce Hox gene activation, which in turn induce leukaemia via aberrant transcription.

Funding: Authors acknowledge financial support from Council of Scientific and Industrial Research (CSIR), India. TP acknowledges research fellowship from CSIR.

Acknowledgements: We acknowledge Yacine Graba for the UAS lines used, Ravindra Chakravarthi, C Subbalakshmi, Aprotim Majumder and Kasavan S for access to and help with imaging facilities.

\section{References:}

1 Hartenstein, V. (2006) Blood cells and blood cell development in the animal kingdom. Annu. Rev. Cell Dev. Biol. 22, 677-712

2 Gold, K.S. and Brückner, K. (2015) Macrophages and cellular immunity in Drosophila melanogaster. Semin. Immunol. 27, 357-368

3 Lebestky, T. et al. (2000) Specification of Drosophila hematopoietic lineage by conserved transcription factors. Science (80-. ). 288, 146-149 
4 Sorrentino, R.P. et al. (2002) Cellular immune response to parasite infection in the Drosophila lymph gland is developmentally regulated. Dev. Biol. 243, 65-80

5 Rizki, T.M. and Rizki, R.M. (1992) Lamellocyte differentiation in Drosophila larvae parasitized by Leptopilina. Dev. Comp. Immunol. 16, 103-110

6 Sam, S. et al. (1996) The serpent gene is necessary for progression through the early stages of fat-body development. Mech. Dev. 60, 197-205

7 Tsai, F.Y. and Orkin, S.H. (1997) Transcription factor GATA-2 is required for proliferation/survival of early hematopoietic cells and mast cell formation, but not for erythroid and myeloid terminal differentiation. Blood 89, 3636-3643

8 Heyworth, C. et al. (1999) A GATA-2/estrogen receptor chimera functions as a liganddependent negative regulator of self-renewal. Genes Dev. 13, 1847-1860

9 Weiss, M.J. and Orkin, S.H. (1995) Transcription factor GATA-1 permits survival and maturation of erythroid precursors by preventing apoptosis. Proc. Natl. Acad. Sci. USA 92, 9623-9627

10 Visvader, J.E. et al. (1992) GATA-1 but not SCL induces megakaryocytic differentiation in an early myeloid line. EMBO J. 11, 4557-4564

11 Kulessa, H. et al. (1995) GATA-1 reprograms avian myelomonocytic cell lines into eosinophils, thromboblasts, and erythroblasts. Genes Dev. 9, 1250-1262

12 Rehorn, K.P. et al. (1996) A molecular aspect of hematopoiesis and endoderm development common to vertebrates and Drosophila. Development 122, 4023-4031

13 Tsang, A.P. et al. (1998) Failure of megakaryopoiesis and arrested erythropoiesis in mice lacking the GATA-1 transcriptional cofactor FOG. Genes Dev. 12, 1176-1188

14 Tsang, A.P. et al. (1997) FOG, a multitype zinc finger protein, acts as a cofactor for transcription factor GATA-1 in erythroid and megakaryocytic differentiation. Cell 90, 109-119

15 Querfurth, E. et al. (2000) Antagonism between C/EBPbeta and FOG in eosinophil lineage commitment of multipotent hematopoietic progenitors. Genes Dev. 14, 25152525

16 Fossett, N. et al. (2001) The Friend of GATA proteins U-shaped, FOG-1, and FOG-2 function as negative regulators of blood, heart, and eye development in Drosophila. Proc. Natl. Acad. Sci. USA 98, 7342-7347

17 Varnum-Finney, B. et al. (1998) The Notch ligand, Jagged-1, influences the development of primitive hematopoietic precursor cells. Blood 91, 4084-4091

18 Lebestky, T. et al. (2003) A Serrate-expressing signaling center controls Drosophila hematopoiesis. Genes Dev. 17, 348-353

19 Parganas, E. et al. (1998) Jak2 is essential for signaling through a variety of cytokine receptors. Cell 93, 385-395 
20 Kieslinger, M. et al. (2000) Antiapoptotic activity of Stat5 required during terminal stages of myeloid differentiation. Genes Dev. 14, 232-244

21 Morin-Poulard, I. et al. (2013) The Drosophila JAK-STAT pathway in blood cell formation and immunity. JAKSTAT 2, e25700

22 Peeters, P. et al. (1997) Fusion of TEL, the ETS-variant gene 6 (ETV6), to the receptorassociated kinase JAK2 as a result of $\mathrm{t}(9 ; 12)$ in a lymphoid and $\mathrm{t}(9 ; 15 ; 12)$ in a myeloid leukemia. Blood 90, 2535-2540

23 Luo, H. et al. (2002) The Hopscotch Jak kinase requires the Raf pathway to promote blood cell activation and differentiation in Drosophila. Mol. Genet. Genomics 267, 5763

24 Silverman, N. and Maniatis, T. (2001) NF-kappaB signaling pathways in mammalian and insect innate immunity. Genes Dev. 15, 2321-2342

25 Pearson, J.C. et al. (2005) Modulating Hox gene functions during animal body patterning. Nat. Rev. Genet. 6, 893-904

26 Argiropoulos, B. and Humphries, R.K. (2007) Hox genes in hematopoiesis and leukemogenesis. Oncogene 26, 6766-6776

27 Banreti, A. et al. (2014) Hox proteins mediate developmental and environmental control of autophagy. Dev. Cell 28, 56-69

28 Sánchez-Herrero, E. (2013) Hox targets and cellular functions. Scientifica (Cairo). 2013, 738257

29 Giampaolo, A. et al. (1995) HOXB gene expression and function in differentiating purified hematopoietic progenitors. Stem Cells 13 Suppl 1, 90-105

30 Giampaolo, A. et al. (1994) Key functional role and lineage-specific expression of selected HOXB genes in purified hematopoietic progenitor differentiation. Blood 84, $3637-3647$

31 Kawagoe, H. et al. (1999) Expression of HOX genes, HOX cofactors, and MLL in phenotypically and functionally defined subpopulations of leukemic and normal human hematopoietic cells. Leukemia (08876924) 13,

32 Pineault, K.M. and Wellik, D.M. (2014) Hox genes and limb musculoskeletal development. Curr. Osteoporos. Rep. 12, 420-427

33 Sauvageau, G. et al. (1994) Differential expression of homeobox genes in functionally distinct CD34+ subpopulations of human bone marrow cells. Proc. Natl. Acad. Sci. USA 91, 12223-12227

34 Fischbach, N.A. et al. (2005) HOXB6 overexpression in murine bone marrow immortalizes a myelomonocytic precursor in vitro and causes hematopoietic stem cell expansion and acute myeloid leukemia in vivo. Blood 105, 1456-1466

35 Kroon, E. et al. (1998) Hoxa9 transforms primary bone marrow cells through specific collaboration with Meis1a but not Pbx1b. EMBO J. 17, 3714-3725 
36 Thorsteinsdottir, U. et al. (2002) Overexpression of the myeloid leukemia-associated Hoxa9 gene in bone marrow cells induces stem cell expansion. Blood 99, 121-129

37 Sauvageau, G. et al. (1995) Overexpression of HOXB4 in hematopoietic cells causes the selective expansion of more primitive populations in vitro and in vivo. Genes Dev. 9, $1753-1765$

38 Sauvageau, G. et al. (1997) Overexpression of HOXB3 in hematopoietic cells causes defective lymphoid development and progressive myeloproliferation. Immunity 6, 13-22

39 Thorsteinsdottir, U. et al. (1997) Overexpression of HOXA10 in murine hematopoietic cells perturbs both myeloid and lymphoid differentiation and leads to acute myeloid leukemia. Mol. Cell. Biol. 17, 495-505

40 Lo, P.C.H. et al. (2002) Homeotic genes autonomously specify the anteroposterior subdivision of the Drosophila dorsal vessel into aorta and heart. Dev. Biol. 251, 307-319

41 Benmimoun, B. et al. (2015) The EBF transcription factor Collier directly promotes Drosophila blood cell progenitor maintenance independently of the niche. Proc. Natl. Acad. Sci. USA 112, 9052-9057

42 Evans, C.J. et al. (2014) Drosophila hematopoiesis: Markers and methods for molecular genetic analysis. Methods 68, 242-251

43 Petraki, S. et al. (2015) Assaying Blood Cell Populations of the Drosophila melanogaster Larva. J. Vis. Exp. DOI: 10.3791/52733

44 Pastor-Pareja, J.C. et al. (2008) An innate immune response of blood cells to tumors and tissue damage in Drosophila. Dis. Model. Mech. 1, 144-54; discussion 153

45 Arefin, B. et al. (2017) The immune phenotype of three drosophila leukemia models. G3 (Bethesda). 7, 2139-2149

46 Singh, N.P. and Mishra, R.K. (2014) Role of abd-A and Abd-B in development of abdominal epithelia breaks posterior prevalence rule. PLoS Genet. 10, e1004717

47 Armstrong, S.A. et al. (2002) MLL translocations specify a distinct gene expression profile that distinguishes a unique leukemia. Nat. Genet. 30, 41-47

48 Ferrando, A.A. et al. (2003) Gene expression signatures in MLL-rearranged T-lineage and B-precursor acute leukemias: dominance of HOX dysregulation. Blood 102, 262268

$49 \mathrm{Xu}$, F. et al. (2016) Genomic loss of EZH2 leads to epigenetic modifications and overexpression of the HOX gene clusters in myelodysplastic syndrome. Oncotarget 7 , 8119-8130

50 Cancer Genome Atlas Research Network et al. (2013) Genomic and epigenomic landscapes of adult de novo acute myeloid leukemia. N. Engl. J. Med. 368, 2059-2074 


\section{Figure Legends:}

Fig.1. A) Larvae with subcutaneous tumors. $D f d, U b x, a b d-A$ and $A b d-B$, when expressed under the drivers $\mathrm{cg}$, $\mathrm{He}$ and $\mathrm{Hml}$ lead to melanised bodies in the viscera. B) The size and penetrance of these bodies was maximum when expressed under $c g$. While tumors do manifest when $\mathrm{He}$ and $\mathrm{Hml}$ are used, they are much rarer and smaller. Expression under Lsp2-Gal4 does not lead to the formation of such bodies.

Fig.2. A) Quantification of blood cells. When Dfd, Ubx, abd-A and Abd-B are driven in blood cells under $\mathrm{cg}$-Gal4, $\mathrm{He}$-Gal4 or $\mathrm{Hml}$-Gal4, but not in the fatbody (Lsp2-Gal4) there is an increase in the overall cell number. B) PhosphoHistone3+ nuclei appear when these genes are expressed in the blood cells, indicating that some of the increase in cell number maybe due to cell autonomous proliferation.

Fig.3. Myospheroid staining for lamellocytes. When $D f d, U b x, a b d-A$ but not $A b d-B$ are driven in blood cells (under $\mathrm{cg}$-Gal4, $\mathrm{He}$-Gal4 or $\mathrm{Hml}$-Gal4), but not in the fatbody (Lsp2-Gal4), large, dorsoventrally flattened cells begin to appear in circulation. These stain positive for mys. Some circulating plasmatocytes also appear to mys+. This indicates that they might be in the process of differentiating.

Fig.4. Percentage of pupal lethality, indicated by larvae that fail to eclose. While diving the genes $D f d, U b x, a b d-A$ and $A b d-B$ with $c g$-Gal4 does cause lethality, so does expressing them in the fatbody under Lsp2-Gal4. Driving these genes in the blood cells ( $\mathrm{He}$-Gal4 and $\mathrm{Hml}$-Gal4) leads to a much lower penetrance of this phenotype. 


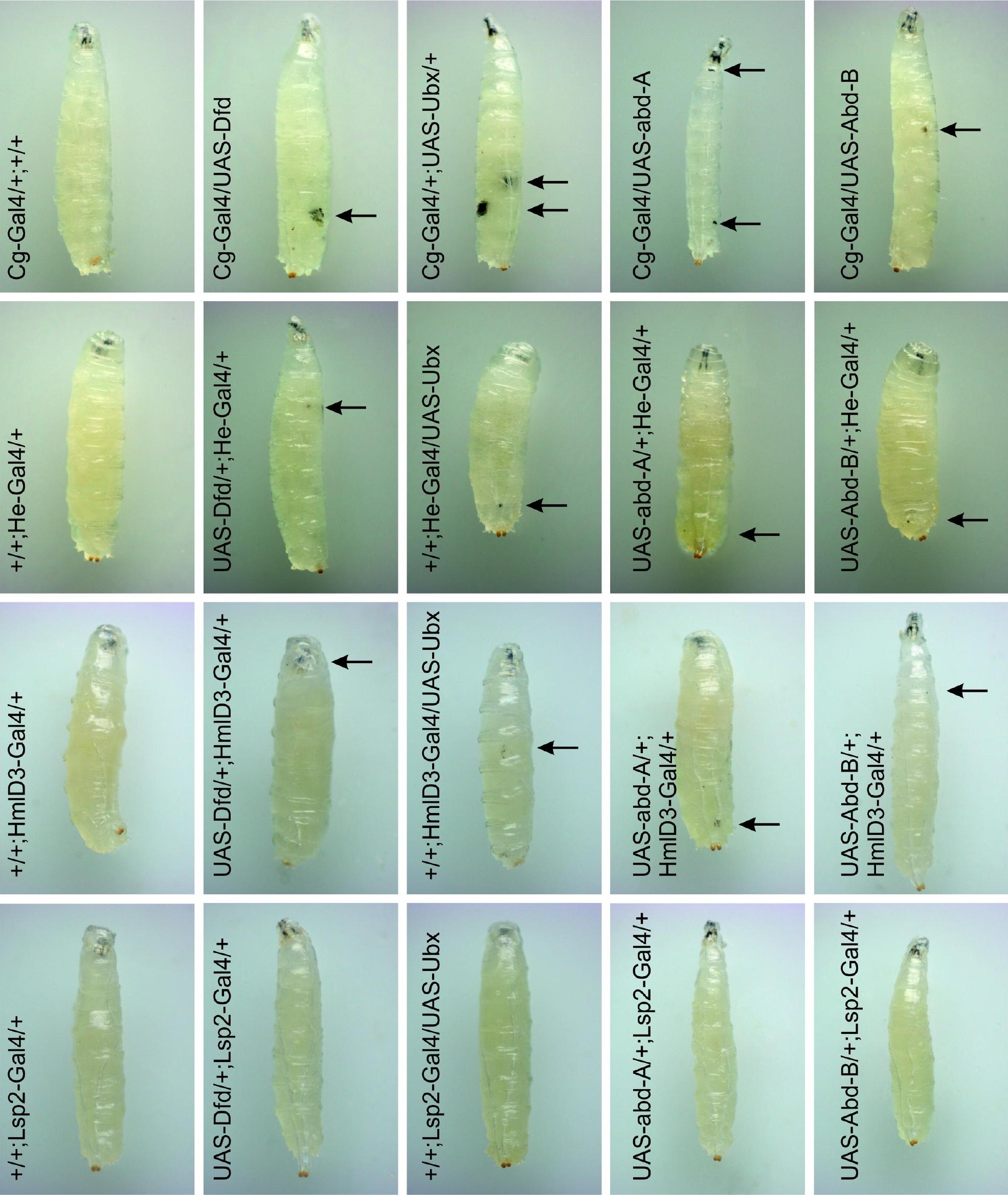




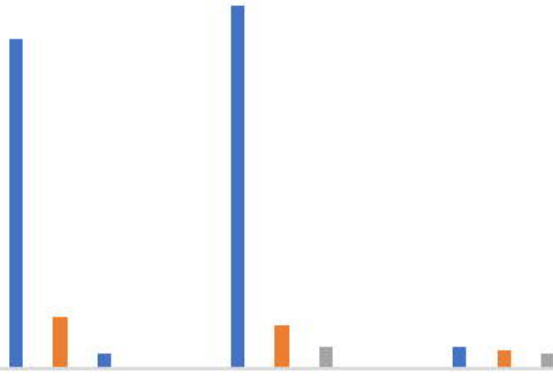

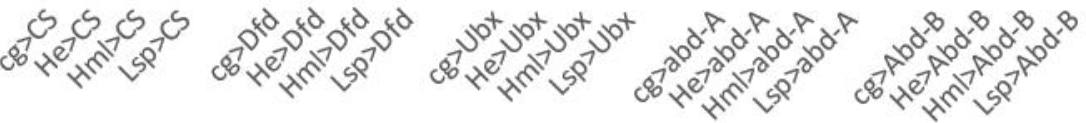


Figure 2B :

DAPI

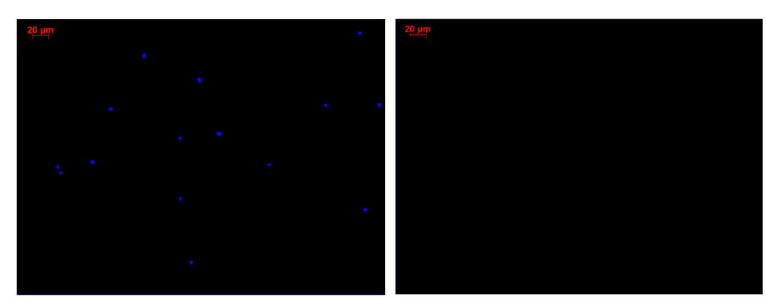

Cg-Gal4/+;+/+

Cg-Gal4/UAS-abd-A

+/+;He-Gal4/+

UAS-abd-A/+;He-Gal4/+

+/+;HmID3-Gal4/+

UAS-abd-A/+;HmID3-Gal4/+

+/+;Lsp2-Gal4/+

UAS-abd-A/+;Lsp2-Gal4/+

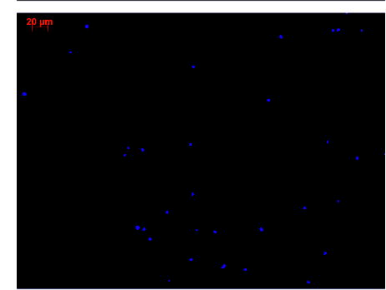

Phalloidin

Merged (+DIC)
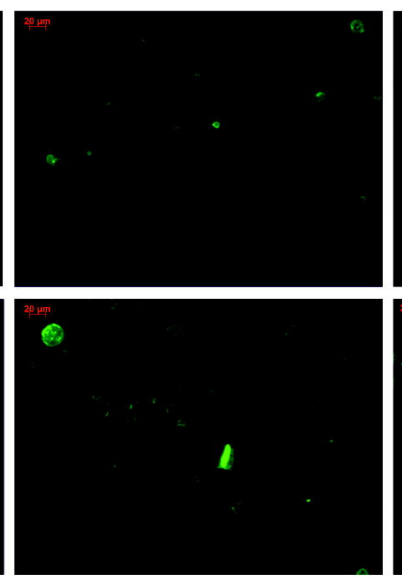

O.

2

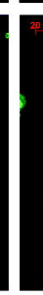

मू

3
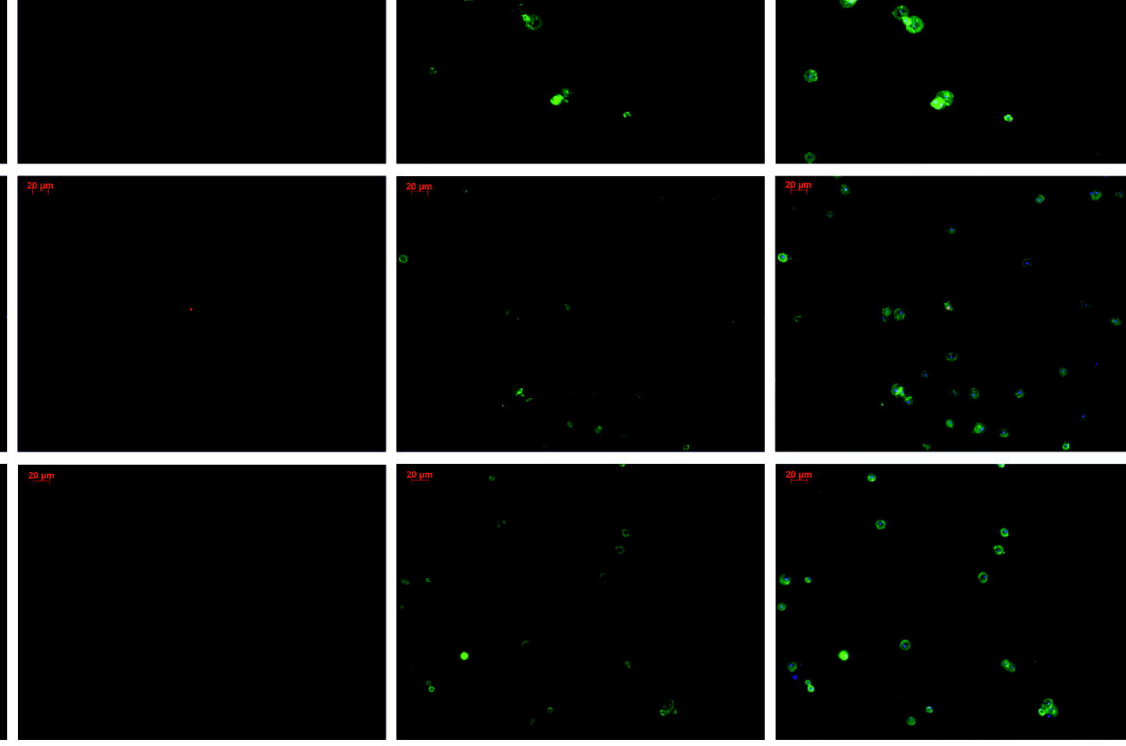

2

37.

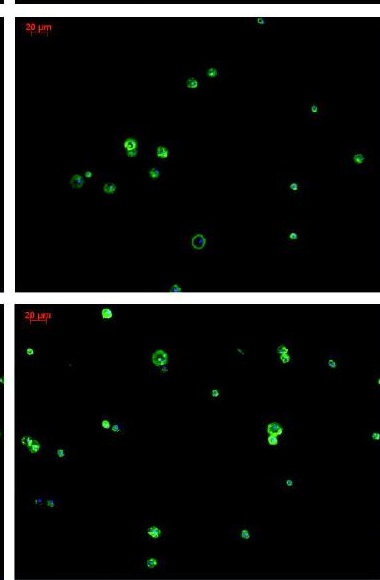




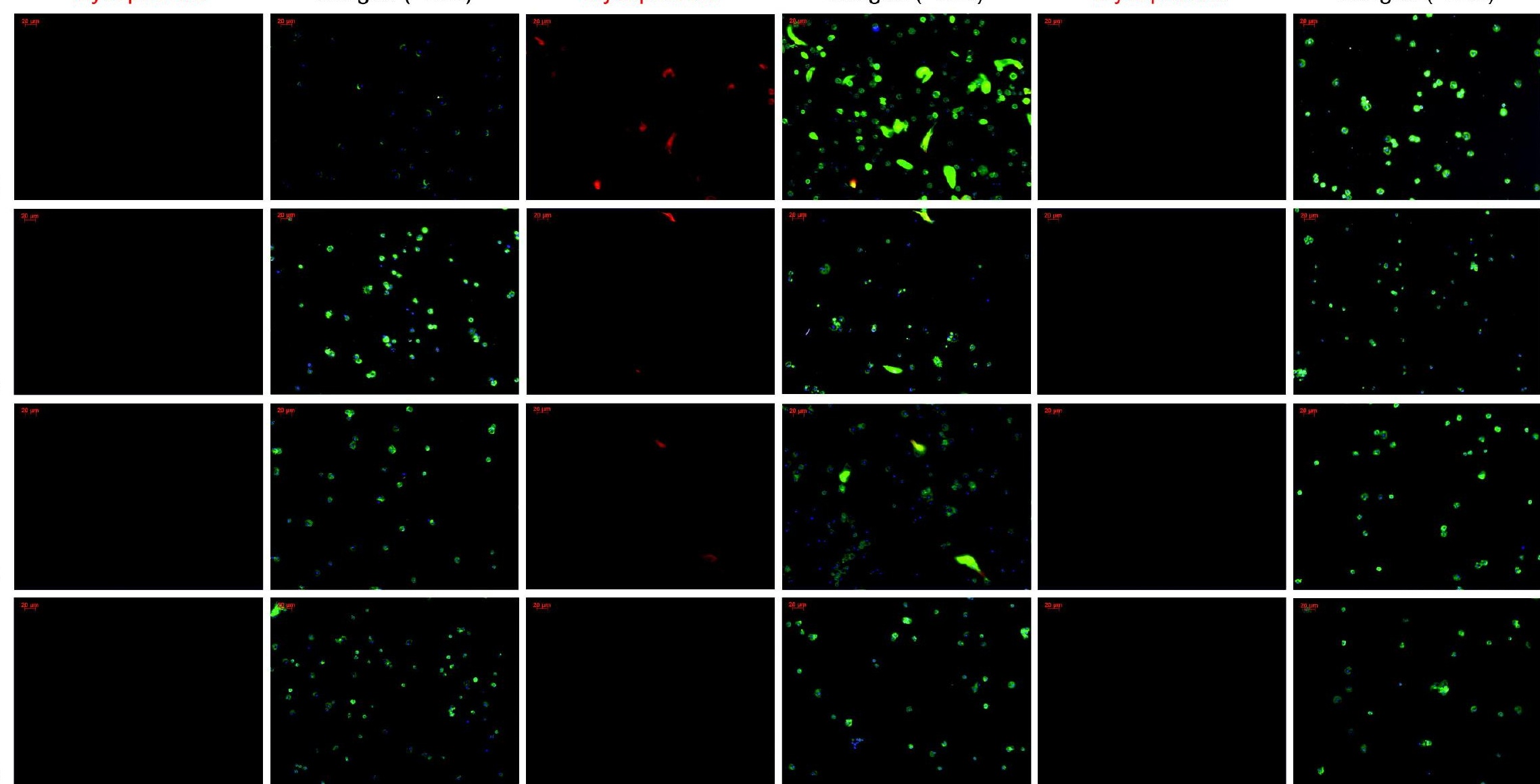


70

60

50

40

30

20

10

0

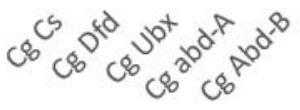

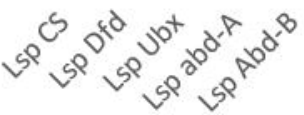

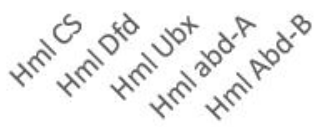

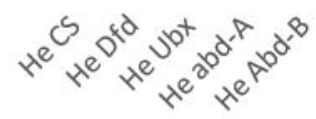

